\title{
Point Rupture Solutions of Singular Elliptic Equations in N-D
}

\author{
Attou Miloua \\ Department of Mathematics \& information Systems CALU, California, PA, 15419 \\ Email: miloua@calu.edu
}

Abstract We consider the elliptic equation

$$
\Delta u=f(u)
$$

in a region $\Omega \subset \mathbb{R}^{N}, N \geq 3$, where $f$ is a positive continuous function satisfying

$$
\lim _{u \rightarrow 0^{+}} f(u)=\infty .
$$

Motivated by the thin film equations, a solution $u$ is said to be a point rupture solution if for some $p \in \Omega, u(p)=0$ and $u(p)>0$ in $\Omega \backslash\{p\}$. Solving the associated ordinary differential equations confirm our main results of sufficient conditions on $f$ for the existence uniquness of radial point rupture solution and its asymptotic behavior. Furthermore, we can prove that our results can be applied to the point rupture solutions for a class of quasi-linear elliptic equations of the form

$$
\operatorname{div}(a(u) \Delta u)=\frac{a^{\prime}(u)}{2}|\nabla u|^{2}+f(u)
$$

Keywords: Thin film; point rupture; radial solution; singular equation; quasi-linear elliptic equation

\section{Introduction}

Let $\Omega$ be a region in $\mathbb{R}^{N}, N \geq 3$ and $f$ be a positive continuous function defined on $(0, \infty)$ such that it can be written as a product of two positive, continuous functions $f_{1}$ and $f_{2}$ such that $f_{1}$ is uniformly bounded and $f_{2}$ is decreasing near zero having single zero at some positive $t_{0}$ and we require $f$ to satisfy the usual growth condition,

$$
\lim _{v \rightarrow 0^{+}} f(v)=\infty
$$

We are interested in the elliptic equation

$$
\Delta u=f(u) \text { in } \Omega
$$

This equation was studied by many authors and its rupture solution was derived in [13] when dealing with the zero set of sobolev functions having negative power of integrability. The same equation was also investigated for its rupture solution in [15] for the case $f(u)=u^{-\alpha}-1$, for $\alpha>1$, and in this case there are many applications to the Van der Waals force driven thin films. The equation was also used in [14], when $f$ satisfies a special integrability condition, and in [11] when the space dimension is 3 and above. The quasi-linear equation (1.4) which can be transformed to (1.2) was analyzed for rupture solutions in [6] where they proved existence of solutions called explosive solutions.

In this we consider $\Omega$ to be a bounded smooth region in $\mathbb{R}^{N}$ where $N \geq 3$ and we assume that $f$ is a positive, continuous function that can be written as a product of two positive, continuous functions $f_{1}$ and $f_{2}$ such that $f_{1}$ is uniformly bounded and $f_{2}$ is decreasing near zero having single zero at some positive $t_{0}$ and we require $f$ to satisfy the usual growth condition. Clearly, $f_{2}$ inherits all the properties of $f$ near zero, more precisely, it will be decreasing, positive, continuous and the same limit as $f$ near zero. Of course our main interest comes from the general $N$ dimensional elliptic equation

$$
u_{t}=-\nabla \cdot\left(u^{m} \nabla u\right)-\nabla \cdot\left(u^{n} \nabla \Delta u\right) .
$$

The second term on the right which is the fourth-order term of the equation reflects surface tension effects, and the second-order term may reflect van der Waals interactions, gravity, the geometry of the 
solid substrate or thermocapillary effects. This class of model equation is related to many physical systems involving fluid interfaces.

When $n=1, m=1$, it describes a thin jet in a Hele-Shaw cell [1], [5], [8], [9]; when $n=m=3$ it describes fluid droplets hanging from a ceiling [10]; when $n=0$ and $m=1$, it describes solidification of a hyper-cooled melt [3], [4]; and when $n=3, m=-1$, it models van der Waals force driven thin film [7], [12], [18], [19], [20], when the space dimension is one R. Laugesen and M. Pugh [16] studied rigorously, in a general setting, positive periodic steady states and touchdown steady states solution. F. Bernis and A. Friedman in [2] established the existence of weak solutions and showed that the support of the thin film will expand with time. Equation (1.3) models the dynamic of thin films equation, using the pressure as defined earlier with Neumann boundary condition $\frac{\partial u}{\partial n}=0$ on $\partial \Omega$. The assumptions of the wetting and non-wetting of surfaces and steady states solutions lead to the semi-linear elliptic equation (1.2). The main result guarantees the existence of a weak radial point rupture solution then this result obtained for the equation (1.2) is exploited to prove the existence of weak point rupture solutions for the quasi-linear elliptic equations of the form

$$
\operatorname{div}(a(u) \nabla u)=\frac{a^{\prime}(u)}{2}|\nabla u|^{2}+f(u)
$$

where for some $\sigma^{*}>0 \quad a \in C^{1}\left[0, \sigma^{*}\right]$ and $f \in C\left(0, \sigma^{*}\right]$ are positive functions of a real variable. Therefore, We are dealing with a semi-linear elliptic equation in $\mathbb{R}^{N}$, and as in the preceding, in $\mathbb{R}^{N}$ for now, let $\Omega$ be a smooth region in $\mathbb{R}^{N}$ with $N \geq 3$ and $f$ be a positive continuous function defined on $(0, \infty)$ satisfying the growth condition (1.1). Moreover we assume the function $f$ to be the product of two functions as mentioned above. Here also, a solution to (1.2) is said to be an $N$ dimensional point rupture solution if for some $p \in \Omega \subset \mathbb{R}^{N}, u(p)=0$ and $u(x)>0$ for any $x \in \Omega \backslash\{p\}$. The main purpose is to find a sufficient condition on the growth of $f$ near the origin so that (1.2) has a radial point rupture solution in $\mathbb{R}^{N}$. The main difficulty of the problem is the same as in the plane, two types of singularities involved. In radial coordinates, $\Delta u=u_{r r}+\frac{1}{r} u_{r}$, becomes singular when $r=0$. Such singularity is artificial if $u$ behaves nicely and $\frac{1}{r} u_{r}$ becomes continuous at $r=0$. However, for rupture solutions, $u_{r}$ itself could blow up at $r=0$. Singularity also arises when we assume (1.1) and the solution touches zero.

\section{The Main Result}

The following is the statement of the main theorem where we assert the existence of an $N$ dimensional point rupture and weak solution to the semi-linear elliptic equation with its appropriate bounds.

Theorem 1. Let $t_{1}>0$ be such that $f$ is a continuous, positive function and can be written as $f=f_{1} f_{2}$ in $\left(0, t_{1}\right]$. Assume that $f_{2}$ is continuous,positive and monotone decreasing, also $f_{1}$ is uniformly bounded, that is, there exist positive constants $A$ and $B$ such that, $A \leq f_{1} \leq B$. The function $f$ is supposed to satisfy the usual blow up condition near zero, that $i s, \lim _{v \rightarrow 0^{+}} f(v)=\infty$., then define:

$$
G(v)=\int_{0}^{v} \frac{1}{f_{2}(s)} d s
$$

Then there exists $r^{*}>0$ and a radial point rupture solution $u_{0}$ to (1.2) in $B_{r^{*}}(0)$ such that $u_{0}=u_{0}(r)$ is continuous on $\left[0, r^{*}\right]$,

$$
u_{0}(0)=0, u_{0}(r)>0 \text { for any } r \in\left(0, r^{*}\right]
$$

and $u_{0}$ is a weak solution to (1.2) in $B_{r^{*}}(0)$. Moreover, $u_{0}$ is monotone increasing and satisfies the following bounds

$$
G^{-1}\left(\frac{A r^{2}}{2 N}\right) \leq u_{0}(r) \leq \frac{B N}{A(N-2)} G^{-1}\left(\frac{A r^{2}}{2 N}\right)
$$




\section{Proof of the Main Result}

Proof. In this part we assume that $f=f_{1} f_{2}$ with $f_{1}$ uniformly bounded and $f_{2}$ is decreasing near zero. so we can fix $t_{1}>0$ so that $f_{2}$ is decreasing in $\left(0, t_{1}\right]$, this is possible since $f_{2}$ is assumed to be decreasing near zero, now for any $\alpha \in\left(0, t_{1}\right]$ define $r_{1}(\alpha)=\inf \left\{r>0, u_{\alpha}(r)=t_{1}\right\}$ and note that such $r_{1}$ exists because of the oscillation of $u_{\alpha}$. Since $f>0$ in $\left(0, t_{1}\right]$ and $u_{\alpha}(0)=\alpha$, we have that $u_{\alpha}$ is increasing in $\left(0, r_{1}\right]$ from $\alpha$ to $t_{1}$ since we have

$$
r^{N-1} u_{\alpha}^{\prime}(r)=\int_{0}^{r} f_{1}\left(u_{\alpha}(s)\right) f_{2}\left(u_{\alpha}(s)\right) s^{N-1} d s .
$$

Therefore, since $f_{2}$ is decreasing in $\left(0, r_{1}\right]$, using the uniform bound of the function $f_{1}$, that is, $A \leq f_{1} \leq B$ we get,

$$
r^{N-1} u_{\alpha}^{\prime}(r) \geq A \int_{0}^{r} f_{2}\left(u_{\alpha}(s)\right) s^{N-1} d s \geq A f_{2}\left(u_{\alpha}(r)\right) \int_{0}^{r} s^{N-1} d s .
$$

Hence, since we defined $G(t)=\int_{0}^{t} \frac{1}{f_{2}(s)} d s \quad$ for $\quad t<t_{0}$ by noting that $G$ is increasing in $\left[0, t_{0}\right]$ we conclude that

$$
\begin{aligned}
& \int_{0}^{r} \frac{u_{\alpha}^{\prime}(s)}{f_{2}\left(u_{\alpha}(s)\right)} d s \geq \int_{0}^{r} \frac{A s}{N} d s, \text { thus } G\left(u_{\alpha}(r)\right) \geq \frac{A r^{2}}{2 N}+G(\alpha) \geq \frac{A r^{2}}{2 N} \\
& u_{\alpha}(r) \geq G^{-1}\left(\frac{A r^{2}}{2 N}\right) \quad \text { for any } \quad \alpha \in\left(0, t_{1}\right), \quad \text { and for all } r \in\left[0, r_{1}\right] .
\end{aligned}
$$

Now we will prove that there exists a constant $r^{*}>0 \quad$ such that $r_{1}(\alpha) \geq r^{*}$ for all small $\alpha$,

Corollary 1. There exists an $r^{*}>0$ such that for any $\alpha \in\left(0, \frac{t_{1}}{2}\right]$

$$
r_{1}(\alpha) \geq r^{*}
$$

We may define,

$$
r^{*}=\sqrt{\frac{2 N}{A} G\left(\frac{A t_{1}}{6 B}\right)}
$$

Proof.

$$
u_{\alpha}^{\prime}(r) \leq B r^{1-N} \int_{0}^{r} f_{2}\left(u_{\alpha}(s)\right) s^{N-1} d s \leq B r^{1-N} \int_{0}^{r} f_{2}\left(G^{-1}\left(\frac{A s^{2}}{2 N}\right)\right) s^{N-1} d s \text { for } r \leq r_{1}
$$

Thus integrating from 0 to $r_{1}$ we get,

$$
t_{1}-\alpha \leq \int_{0}^{r_{1}} B r^{1-N} \int_{0}^{r} f_{2}\left(G^{-1}\left(\frac{A s^{2}}{2 N}\right)\right) s^{N-1} d s d r
$$

interchanging integration we get

$$
t_{1}-\alpha \leq B \int_{0}^{r_{1}} f_{2}\left(G^{-1}\left(\frac{A s^{2}}{2 N}\right)\right) s^{N-1} \int_{s}^{r_{1}} r^{1-N} d r d s
$$

hence

$$
t_{1}-\alpha \leq \frac{B}{N-2} \int_{0}^{r_{1}} f_{2}\left(G^{-1}\left(\frac{A s^{2}}{2 N}\right)\right) s d s
$$


Using the change of variable $z=\frac{A s^{2}}{2 N} \quad$ we get

$$
t_{1}-\alpha \leq \frac{B N}{A(N-2)} \int_{0}^{\frac{A r_{1}^{2}}{2 N}} f_{2}\left(G^{-1}(z)\right) d z=\frac{B N}{A(N-2)} G^{-1}\left(\frac{A r_{1}^{2}}{2 N}\right)
$$

So for $\quad 0<\alpha<\frac{t_{1}}{2} \quad$ we get,

$$
\frac{A r_{1}^{2}}{2 N} \geq G\left(\frac{A(N-2) t_{1}}{B 2 N}\right) \geq G\left(\frac{A t_{1}}{6 B}\right)
$$

Therefore $r_{1}(\alpha)$ is uniformly bounded from below for any $\alpha>0$ small enough.

Take $r^{*}$ to be $r^{*}=\sqrt{\frac{2 N}{A} G\left(\frac{A t_{1}}{6 B}\right)}$.

Now, observe that for any $\alpha<\delta<r \leq r^{*}$ we have

$$
G^{-1}\left(\frac{A r^{2}}{2 N}\right) \leq u_{\alpha}(r) \leq t_{1} \leq \frac{B N}{A(N-2)} G^{-1}\left(\frac{A r_{1}^{2}}{2 N}\right)+\alpha .
$$

Using standard elliptic theory and diagonal process we can construct a subsequence $u_{\alpha}$ that converges locally and uniformly to the rupture solution $u_{0}$ that satisfies $\Delta u_{0}=f\left(u_{0}\right)$.

Proposition 1. There exists a sequence $\left\{\alpha_{k}\right\}_{k=1}^{\infty} \subset\left(0, \frac{t_{1}}{2}\right]$ satisfying

$$
\lim _{k \rightarrow \infty} \alpha_{k}=0
$$

such that $u_{\alpha_{k}} \rightarrow u_{0}$ uniformly in $\overline{B_{r^{*}}(0)}$ as $k \rightarrow \infty$, for some function

$$
u_{0} \in C^{0}\left(\overline{B_{r^{*}}(0)}\right) \cap C^{2}\left(\overline{B_{r^{*}}(0)} \backslash\{0\}\right) .
$$

Moreover, $u_{0}$ is a classical solution to (1.2) in $B_{r^{*}}(0) \backslash\{0\}$ and

$$
G^{-1}\left(\frac{A r^{2}}{2 N}\right) \leq u_{0}(r) \leq \frac{B N}{A(N-2)} G^{-1}\left(\frac{A r^{2}}{2 N}\right)
$$

Proof. For any $\varepsilon>0, u_{\alpha}, \alpha \in\left(0, \frac{t_{1}}{2}\right]$ is a family of uniformly bounded classical solutions to

$$
\Delta u=f(u) \text { in } \overline{B_{r^{*}}(0)} \backslash B_{\varepsilon}(0),
$$

hence by a diagonal argument, there exists a sequence $\left\{\alpha_{k}\right\}_{k=1}^{\infty} \subset\left(0, \frac{t_{1}}{2}\right]$ satisfying $\lim _{k \rightarrow \infty} \alpha_{k}=0$, such that $u_{\alpha_{k}} \rightarrow u_{0}$ locally uniformly in $\overline{B_{r^{*}}(0)} \backslash\{0\}$ as $k \rightarrow \infty$. Now (3.1) implies

$$
G^{-1}\left(\frac{A r^{2}}{2 N}\right) \leq u_{0}(r) \leq \frac{B N}{A(N-2)} G^{-1}\left(\frac{A r^{2}}{2 N}\right)
$$

Since

$$
\lim _{r \rightarrow 0} \frac{B N}{A(N-2)} G^{-1}\left(\frac{A r^{2}}{2 N}\right)=0
$$

it is not difficult to see, from the bounds of $u_{\alpha}$ and $u_{0}$, that $u_{\alpha_{k}} \rightarrow u_{0}$ uniformly in $\overline{B_{r^{*}}(0)}$ as $k \rightarrow \infty$.

Remark 1. The above limit should be independent of the choice of the sequence $\left\{\alpha_{k}\right\}_{k=1}^{\infty}$. Actually, we expect that $u_{\alpha} \rightarrow u_{0}$ uniformly on $\left[0, r^{*}\right]$ as $\alpha \rightarrow 0$. This is an open question. 
In order to show that $u_{0}$ is a weak solution. The following lemma will be very useful.

Lemma 1. Let $v_{0}$ be the rupture radial solution in $\mathbb{R}^{n}$ of,

$$
\Delta v=h(v) \quad \text { then } \quad \lim _{r \rightarrow 0^{+}} r^{n-1} v_{0}^{\prime}(r)=0 .
$$

Proof. We have the followings,

$$
\begin{gathered}
\Delta v_{0}=h\left(v_{0}\right), \quad \text { since radial in } \mathbb{R}^{n}, \quad v_{0}^{\prime \prime}+\frac{n-1}{r} v_{0}^{\prime}=h\left(v_{0}\right) \\
r^{n-1} v_{0}^{\prime \prime}+(n-1) r^{n-2} v_{0}^{\prime}(r)=r^{n-1} h\left(v_{0}\right) \quad \text { or }\left(r^{n-1} v_{0}^{\prime}\right)^{\prime}=r^{n-1} h\left(v_{0}\right)>0
\end{gathered}
$$

Hence, we see that $r^{n-1} v_{0}^{\prime}(r)$ is monotone increasing in $\left(0, r^{*}\right)$. Since $r^{n-1} v_{0}^{\prime} \geq 0$ in $\left(0, r^{*}\right)$

$$
\beta=\lim _{r \rightarrow 0^{+}} r^{n-1} v_{0}^{\prime}(r) \geq 0 .
$$

is well defined. Assuming that $\beta>0$, there exists $\delta>0$ such that for $r \in(0, \delta]$ we have,

$$
r^{n-1} v_{0}^{\prime}(r) \geq \frac{\beta}{2} \text { thus we get } v_{0}(r) \leq v_{0}(\delta)-\frac{\beta \delta^{2-n}}{2-n}+\frac{\beta}{2(2-n) r^{n-2}}, \text { which is a contradiction. }
$$

Now, in $\mathbb{R}^{n}$ the rupture solution for, $\Delta v=h(v)$ is a weak solution in $\Omega=B_{r^{*}}(0)$.

Proposition 2. $h\left(v_{0}\right) \in L^{1}(\Omega)$ and $v_{0}$ is a weak solution to, $\Delta v=h(v)$.

Proof. For any test function $\varphi \in C_{c}^{\infty}(\Omega)$, we have

$$
\begin{aligned}
& \int_{\Omega} v_{0} \Delta \varphi d x=\lim _{\varepsilon \rightarrow 0^{+}} \int_{\Omega \backslash \overline{B_{\varepsilon}(0)}} v_{0} \Delta \varphi d x \\
& =\lim _{\varepsilon \rightarrow 0^{+}}\left(\int_{\Omega \backslash \overline{B_{\varepsilon}(0)}} \Delta v_{0} \varphi d x-\int_{\partial B_{\varepsilon}(0)}\left(v_{0} \frac{\partial \varphi}{\partial n}-\varphi \frac{\partial v_{0}}{\partial n}\right) d s_{x}\right) \\
& =\lim _{\varepsilon \rightarrow 0^{+}}\left(\int_{\Omega \backslash \frac{B_{\varepsilon}(0)}{}} h\left(v_{0}\right) \varphi d x-\int_{\partial B_{\varepsilon}(0)} v_{0} \frac{\partial \varphi}{\partial n} d s_{x}+\int_{\partial B_{\varepsilon}(0)} \varphi \frac{\partial v_{0}}{\partial n} d s_{x}\right) .
\end{aligned}
$$

Now for any $\varepsilon \in\left(0, r^{*}\right)$, since $v_{0}(\varepsilon) \leq v_{0}\left(r^{*}\right) \leq t_{1}$, we have

$$
\begin{aligned}
\left|\int_{\partial B_{\varepsilon}(0)} v_{0} \frac{\partial \varphi}{\partial n} d s_{x}\right| & \leq v_{0}(\varepsilon)\|\nabla \varphi\|_{L^{\infty}(\Omega)}\left|\partial B_{\varepsilon}(0)\right| \\
& \leq n \alpha(n) \varepsilon^{n-1} v_{0}(\varepsilon)\|\nabla \varphi\|_{L^{\infty}\left(B_{r^{*}}(0)\right)} \rightarrow 0
\end{aligned}
$$

as $\varepsilon \rightarrow 0^{+}$. Here $n \alpha(n)$ denotes the surface area of the unit sphere. On the other hand, the previous lemma implies that

$$
\left|\int_{\partial B_{\varepsilon}(0)} \varphi \frac{\partial v_{0}}{\partial n} d s_{x}\right| \leq n \alpha(n) \varepsilon^{n-1} v_{0}^{\prime}(\varepsilon)\|\varphi\|_{L^{\infty}\left(B_{r^{*}}(0)\right)} \rightarrow 0
$$


as $\varepsilon \rightarrow 0^{+}$. Hence, we have for any $\varphi \in C_{c}^{\infty}(\Omega)$,

$$
\int_{B_{r^{*}}(0)} v_{0} \Delta \varphi d x=\lim _{\varepsilon \rightarrow 0^{+}} \int_{B_{r^{*}}(0) \backslash \overline{B_{\varepsilon}(0)}} h\left(v_{0}\right) \varphi d x .
$$

Choosing $\varphi$ such that $\varphi \equiv 1$ near the origin, the above limit implies that $h\left(v_{0}\right)$ is integrable near the origin. Since $h\left(v_{0}\right)$ is a positive continuous function in $B_{r^{*}}(0) \backslash\{0\}$, we conclude $h\left(v_{0}\right) \in L^{1}\left(B_{r^{*}}(0)\right)$. So we have for any test function $\varphi \in C_{c}^{\infty}\left(B_{r^{*}}(0)\right)$

$$
\int_{B_{r^{*}}(0)} v_{0} \Delta \varphi d x=\lim _{\varepsilon \rightarrow 0^{+}} \int_{B_{r^{*}}(0) \backslash \overline{B_{\varepsilon}(0)}} h\left(v_{0}\right) \varphi d x=\int_{B_{r^{*}}(0)} h\left(v_{0}\right) \varphi d x
$$

i.e., $v_{0}$ is a weak solution in $B_{r^{*}}(0) \subseteq \mathbb{R}^{n}$.

\section{Application to Quasi-Linear Equations}

Our previous result can easily be applied in $\mathbb{R}^{n}$ to the point rupture solutions of the quasi-linear elliptic equations of the form

$$
\operatorname{div}(a(u) \Delta u)=\frac{a^{\prime}(u)}{2}|\nabla u|^{2}+f(u)
$$

Now let us state the theorem for an $n$ dimensional quasi-linear elliptic equation.

Theorem 2. Assume that for some $\sigma^{*}>0, a \in C^{1}\left[0, \sigma^{*}\right] f \in C\left(0, \sigma^{*}\right]$ are positive functions. Let $f_{1}, f_{2}$ be continuous, $f_{2}$ is monotone decreasing $f_{1}$ is uniformly bounded, such that

$$
\lim _{v \rightarrow 0^{+}} f_{2}(v)=\infty, \quad \text { and } \quad f=f_{1} f_{2}
$$

Let

$$
G(v)=\int_{0}^{v} \frac{1}{f_{2}(s)} d s
$$

Then there exists $r^{*}>0$ and a radial point rupture solution $u_{0}$ to (1.4) in $B_{r^{*}}(0)$ such that $u_{0}=u_{0}(r)$ is continuous on $\left[0, r^{*}\right]$,

$$
u_{0}(0)=0, u_{0}(r)>0 \text { for any } r \in\left(0, r^{*}\right] .
$$

Moreover there exists a function $g$, and constants $K_{1}, K_{2}$ such that $u_{0}$ is a weak solution with the following estimates

$$
g\left(G^{-1}\left(\frac{A r^{2}}{2 N}\right)\right) \leq u_{0}(r) \leq g\left(\frac{K_{2} N}{K_{1}(N-2)} G^{-1}\left(\frac{K_{1} r^{2}}{2 N}\right)\right)
$$

Proof. We consider the quasi-linear equation (1.4) in a region $\Omega \subset \mathbb{R}^{n}$ where for some $\delta^{*}>0, a \in C^{1}\left[0, \delta^{*}\right]$ and $f \in C\left(0, \delta^{*}\right]$ are positive functions of a real variable. A solution to (1.4) is said to be a point rupture solution if for some $p \in \Omega, u(p)=0$ and $u(x)>0$ for any $x \in \Omega \backslash\{p\}$. 
Let $g$ be a solution to the Cauchy problem

$$
g^{\prime}=\frac{1}{\sqrt{a(g)}}, g(0)=0,
$$

and let $v$ be a solution to the elliptic problem

$$
\triangle v=h(v)
$$

where

$$
h(v)=\frac{f(g(v))}{\sqrt{a(g(v))}} .
$$

Define the auxiliary function $u$ as $u=g \circ v$ from $\mathbb{R}^{N}$ to $\mathbb{R}$ which is well defined since $v$ is a solution of (4.3) and $h$ is continuous, then

$$
u=g(v) \text { thus } h(v)=\frac{f(g(v))}{\sqrt{a(g(v))}}=\frac{f(u)}{\sqrt{a(u)}} .
$$

Therefore it is clear that,

$$
\nabla u=g^{\prime}(v) \nabla v
$$

hence,

$$
\nabla u=\frac{1}{\sqrt{a(g(v))}} \nabla v
$$

thus

$$
\nabla u=\frac{1}{\sqrt{a(u)}} \nabla v
$$

Therefore we have

$$
\nabla v=\sqrt{a(u)} \nabla u
$$

On the other hand

$$
\nabla \sqrt{a(u)}=\frac{1}{2}(a(u))^{\frac{-1}{2}} a^{\prime}(u) \nabla u=\frac{a^{\prime}(u)}{2 \sqrt{a(u)}} \nabla u,
$$

Now combining all of the above leads to

$$
\Delta v=\sqrt{a(u)} \Delta u+\frac{1}{2} \frac{1}{\sqrt{a(u)}} a^{\prime}(u)|\nabla u|^{2} .
$$

Hence (4.3) implies

$$
\sqrt{a(u)} \Delta u+\frac{1}{2} \frac{1}{\sqrt{a(u)}} a^{\prime}(u)|\nabla u|^{2}=\frac{f(u)}{\sqrt{a(u)}}
$$

that is,

$$
a(u) \Delta u+\frac{1}{2} a^{\prime}(u)|\nabla u|^{2}=f(u),
$$

and now by adding to both sides the quantity,

$$
\frac{1}{2} a^{\prime}(u)|\nabla u|^{2}
$$

then we end up with,

$$
a(u) \Delta u+a^{\prime}(u)|\nabla u|^{2}=\frac{1}{2} a^{\prime}(u)|\nabla u|^{2}+f(u),
$$


which is equivalent to (1.4). Hence, (1.4) possesses a point rupture solution if and only if (4.3) has a point rupture solution.

Now $h$ can be written as a product of two function satisfying conditions of the previous theorem

$$
h(v)=\frac{f(g(v))}{\sqrt{a(g(v))}}=\frac{f_{1}(g(v))}{\sqrt{a(g(v))}} f_{2}(g(v))=h_{1}(v) h_{2}(v)
$$

where, $h_{1}=\frac{f_{1}(g(v))}{\sqrt{a(g(v))}}$ is uniformly bounded, say $K_{1} \leq K_{2} \quad$ since both $\quad f_{1}$ and $g$ are uniformly bounded. The function $h_{2}$ defined by $h_{2}(v)=f_{2}(g(v))$ is decreasing since $g^{\prime}>0$. Therefore the technical assumptions on $f_{1}$ and $f_{2}$ imply that the function $h$ satisfies the conditions of theorem 5.1. Hence, there exists a weak radial rupture solution $v_{0}$ which is equivalent to say that $u_{0}=g\left(v_{0}\right)$ is a radial rupture solution for the quasi-linear equation with the bounds,

$$
g\left(G^{-1}\left(\frac{A r^{2}}{2 N}\right)\right) \leq u_{0}(r) \leq g\left(\frac{K_{2} N}{K_{1}(N-2)} G^{-1}\left(\frac{K_{1} r^{2}}{2 N}\right)\right)
$$

Now we will prove that in $\mathbb{R}^{n}$ the rupture solution for the quasi-linear equation is a weak solution in $\Omega=B_{r^{*}}(0)$.

Proposition 3. $f\left(u_{0}\right) \in L^{1}(\Omega)$ and $u_{0}$ is a weak solution for the quasi-linear equation in $\Omega=B_{r^{*}}(0)$

Proof. Assume that $u_{0}$ is a rupture solution for the quasi-linear equation, that is $u_{0}$ satisfies,

$$
\operatorname{div}(a(u) \nabla u)=\frac{a^{\prime}(u)}{2}|\nabla u|^{2}+f(u)
$$

where $a \in C^{1}, f \in C^{0}$ are positive functions and $u_{0}(0)=0$.

We need to show that for any test function $\varphi \in C_{c}^{\infty}(\Omega)$, we have

$$
\begin{gathered}
\int_{\Omega} \operatorname{div}\left(a\left(u_{0}\right) \nabla u_{0}\right) \varphi d x=\int_{\Omega}\left(\frac{a^{\prime}\left(u_{0}\right)}{2}\left|\nabla u_{0}\right|^{2}+f\left(u_{0}\right)\right) \varphi d x \\
\int_{\Omega}\left(a\left(u_{0}\right) \Delta u_{0}+\frac{1}{2} a^{\prime}\left(u_{0}\right)\left|\nabla u_{0}\right|^{2}-f\left(u_{0}\right)\right) \varphi d x=0 \quad \text { that is, } \\
\int_{\Omega} \sqrt{a\left(u_{0}\right)}\left[\sqrt{a\left(u_{0}\right)} \Delta u_{0}+\frac{1}{2 \sqrt{a\left(u_{0}\right)}} a^{\prime}\left(u_{0}\right)\left|\nabla u_{0}\right|^{2}-\frac{f\left(u_{0}\right)}{\sqrt{a\left(u_{0}\right)}}\right] \varphi d x=0 \quad \text { thus, } \\
\int_{\Omega}\left[\sqrt{a\left(u_{0}\right)} \Delta u_{0}+\frac{1}{2 \sqrt{a\left(u_{0}\right)}} a^{\prime}\left(u_{0}\right)\left|\nabla u_{0}\right|^{2}-\frac{f\left(u_{0}\right)}{\sqrt{a\left(u_{0}\right)}}\right] \sqrt{a\left(u_{0}\right)} \varphi d x=0 \quad \text { hence, } \\
\int_{\Omega}\left[\Delta v_{0}-h\left(v_{0}\right)\right] \sqrt{a\left(g\left(v_{0}\right)\right)} \varphi d x=0 \\
\int_{\Omega}^{\Delta v_{0} \sqrt{a\left(g\left(v_{0}\right)\right)} \varphi d x=\int_{\Omega} h\left(v_{0}\right)} \sqrt{a\left(g\left(v_{0}\right)\right)} \varphi d x
\end{gathered}
$$

Define $\psi=\sqrt{a\left(g\left(v_{0}\right)\right)} \varphi$ and then let us start computing, 


$$
\begin{aligned}
\int_{\Omega} v_{0} \Delta \psi d x=\lim _{\varepsilon \rightarrow 0^{+}} \int_{\Omega \backslash \overline{B_{\varepsilon}(0)}} v_{0} \Delta \psi d x & =\lim _{\varepsilon \rightarrow 0^{+}}\left[\int_{\Omega \backslash \overline{B_{\varepsilon}(0)}} \psi \Delta v_{0} d x-\int_{\partial B_{\varepsilon}(0)}\left(v_{0} \frac{\partial \psi}{\partial n}-\psi \frac{\partial v_{0}}{\partial n}\right) d s_{x}\right] \\
& =\lim _{\varepsilon \rightarrow 0^{+}}\left[\int_{\Omega \backslash \overline{B_{\varepsilon}(0)}} h\left(v_{0}\right) \psi d x-\int_{\partial B_{\varepsilon}(0)}\left(v_{0} \frac{\partial \psi}{\partial n}-\psi \frac{\partial v_{0}}{\partial n}\right) d s_{x}\right]
\end{aligned}
$$

Now let's justify that the boundary terms are zeros, indeed for any $\varepsilon \in\left(0, r^{*}\right)$ since $v_{0}(\varepsilon) \leq v_{0}\left(r^{*}\right)$, we have

$$
\left|\int_{\partial B_{\varepsilon}(0)} \psi \frac{\partial v_{0}}{\partial n} d s_{x}\right| \leq M n \alpha(n) \varepsilon^{n-1} v_{0}^{\prime}(\varepsilon)\|\varphi\|_{L^{\infty}\left(B_{r^{*}}(0)\right)} \rightarrow 0 \text { as } \varepsilon \rightarrow 0
$$

Here $M$ is a constant and we use the lemma. On the other hand the second boundary term can be controlled as follows,

$$
\left|\int_{\partial B_{\varepsilon}(0)} v_{0} \frac{\partial \psi}{\partial n} d s_{x}\right| \leq\left[\left(M\|\nabla \varphi\|_{L^{\infty}\left(B_{r^{*}}(0)\right)}\right)+\left(N\|\varphi\|_{L^{\infty}\left(B_{r^{*}}(0)\right)}\right) v_{0}^{\prime}(\varepsilon)\right] n \alpha(n) \varepsilon^{n-1}
$$

Where $N=\max _{B_{r^{*}}(0)} \frac{a^{\prime}\left(g\left(v_{0}\right)\right) g^{\prime}\left(v_{0}\right)}{2 \sqrt{a\left(g\left(v_{0}\right)\right)}}$

Therefore we have that,

$$
\left|\int_{\partial B_{\varepsilon}(0)} v_{0} \frac{\partial \psi}{\partial n} d s_{x}\right| \rightarrow 0^{+} \text {as } \varepsilon \rightarrow 0^{+}
$$

Hence, $f\left(u_{0}\right) \in L^{1}(\Omega)$ and $u_{0}$ is a weak solution for the quasi-linear equation in $\Omega=B_{r^{*}}(0)$. 


\section{References}

1. Robert Almgren, Andrea Bertozzi, and Michael P. Brenner. Stable and unstable singularities in the unforced Hele-Shaw cell. Phys. Fluids, 8(6):1356-1370, 1996.

2. Francisco Bernis and Avner Friedman. Higher order nonlinear degenerate parabolic equations. J. Differential Equations, 83(1):179-206, 1990.

3. Andrew J. Bernoff and Andrea L. Bertozzi. Singularities in a modified Kuramoto-Sivashinsky equation describing interface motion for phase transition. Phys. D, 85(3):375-404, 1995.

4. A. L. Bertozzi and M. C. Pugh. Finite-time blow-up of solutions of some long-wave unstable thin film equations. Indiana Univ. Math. J., 49(4):1323-1366, 2000.

5. Andrea L. Bertozzi, Michael P. Brenner, Todd F. Dupont, and Leo P. Kadanoff. Singularities and similarities in interface flows. In Trends and perspectives in applied mathematics, volume 100 of Appl. Math. Sci., pages 155-208. Springer, New York, 1994.

6. Francesca Gladiali and Marco Squassina. On Explosive Solutions for a class of Quasi-linear Elliptic Equations. Advanced Nonlinear Studies, 13:663-698, 2013.

7. Xinfu Chen and Huiqiang Jiang. Singular limit of an energy minimizer arising from dewetting thin film model with van der waal, born repulsion and surface tension forces. Calc. Var. Partial Differential Equations, 44(1-2):221-246, 2012.

8. Peter Constantin, Todd F. Dupont, Raymond E. Goldstein, Leo P. Kadanoff, Michael J. Shelley, and Su-Min Zhou. Droplet breakup in a model of the Hele-Shaw cell. Phys. Rev. E (3), 47(6):4169-4181, 1993.

9. Todd F. Dupont, Raymond E. Goldstein, Leo P. Kadanoff, and Su-Min Zhou. Finite-time singularity formation in Hele-Shaw systems. Phys. Rev. E (3), 47(6):4182-4196, 1993.

10. P. Ehrhard. The spreading of hanging drops. J. Colloid \& Interface, 168(1):242-246, 1994.

11. Zongming Guo, Dong Ye, and Feng Zhou. Existence of singular positive solutions for some semilinear elliptic equations. Pacific J. Math., 236(1):57-71, 2008.

12. Huiqiang Jiang. Energy minimizers of a thin film equation with Born repulsion force. Commun. Pure Appl. Anal., 10(2):803-815, 2011.

13. Huiqiang Jiang and Fanghua Lin. Zero set of soblev functions with negative power of integrability. Chinese Ann. Math. Ser. B, 25(1):65-72, 2004.

14. Huiqiang Jiang and Attou Miloua. Point rupture solutions of a singular elliptic equation. Electronic Journal of Differential Equations, 2013(70):1-8,2013.

15. Huiqiang Jiang and Wei-Ming Ni. On steady states of van der Waals force driven thin film equations. European J. Appl. Math., 18(2):153-180, 2007.

16. R. S. Laugesen and M. C. Pugh. Properties of steady states for thin film equations. European J. Appl. Math., 11(3):293-351, 2000.

17. Michael J. Shelley, Raymond E. Goldstein, and Adriana I. Pesci. Topological transitions in Hele-Shaw flow. In Singularities in fluids, plasmas and optics (Heraklion, 1992), volume 404 of NATO Adv. Sci. Inst. Ser. C Math. Phys. Sci., pages 167-188. Kluwer Acad. Publ., Dordrecht, 1993.

18. B. Williams and S. H. Davis. Nonlinear theory of film rupture. J. Colloid Interface Sci., 90:220-228, 1982.

19. Thomas P. Witelski and Andrew J. Bernoff. Stability of self-similar solutions for van der Waals driven thin film rupture. Phys. Fluids, 11(9):2443-2445, 1999.

20. Wendy W. Zhang and John R. Lister. Similarity solutions for van der Waals rupture of a thin film on a solid substrate. Phys. Fluids, 11(9):2454-2462, 1999. 